\title{
Screening and Characterization of Lactic Acid Bacteria Strains with Anti-inflammatory Activities through in vitro and Caenorhabditis elegans Model Testing
}

\author{
Hye Kyoung Lee, Sun-Hae Choi, Cho Rong Lee, Sun Hee Lee, Mi Ri Park', \\ Younghoon $\mathrm{Kim}^{1}$, Myung-Ki Lee ${ }^{2}$, and Geun-Bae Kim* \\ Department of Animal Science and Technology, Chung-Ang University, Anseong 456-756, Korea \\ ${ }^{1}$ BK21 Plus Graduate Program, Department of Animal Science and Institute Agricultural Science \& Technology, \\ Chonbuk National University, Jeonju 561-756, Korea \\ ${ }^{2}$ Fermentation and Functionality Research Group, Korea Food Research Institute, Sungnam 463-746, Korea
}

\begin{abstract}
The present study was conducted to screen candidate probiotic strains for anti-inflammatory activity. Initially, a nitric oxide (NO) assay was used to test selected candidate probiotic strains for anti-inflammatory activity in cultures of the murine macrophage cell line, RAW 264.7. Then, the in vitro probiotic properties of the strains, including bile tolerance, acid resistance, and growth in skim milk media, were investigated. We also performed an in vitro hydrophobicity test and an intestinal adhesion assay using Caenorhabditis elegans as a surrogate in vivo model. From our screening, we obtained 4 probiotic candidate lactic acid bacteria (LAB) strains based on their anti-inflammatory activity in lipopolysaccharide (LPS)-stimulated RAW 264.7 cell cultures and the results of the in vitro and in vivo probiotic property assessments. Molecular characterization using 16S rDNA sequencing analysis identified the $4 \mathrm{LAB}$ strains as Lactobacillus plantarum. The selected L. plantarum strains (CAU1054, CAU1055, CAU1064, and CAU1106) were found to possess desirable in vitro and in vivo probiotic properties, and these strains are good candidates for further investigations in animal models and human clinical studies to elucidate the mechanisms underlying their anti-inflammatory activities.
\end{abstract}

Key words: lactic acid bacteria, probiotics, anti-inflammatory, Caenorhabditis elegans

\section{Introduction}

Lactic acid bacteria (LAB) are gram-positive organisms that are found throughout nature, in vegetables, meats, dairy products, and many parts of human body, including the gastrointestinal (GI) tract. Probiotics can be defined as "live microbial food ingredients that, when administered in adequate amounts, confer a health benefit on a host" (FAO/WHO, 2001). These beneficial effects include amelioration of lactose intolerance, anti-mutagenic and anti-carcinogenic activities, reduction of serum cholesterol, modulation of immune responses, and anti-inflammatory activities (Maldonado et al., 2007; Mishra and Prasad, 2005). The genera most commonly used in probiotic applications are Lactobacillus, Pediococcus, Weis-

*Corresponding author: Geun-Bae Kim, Department of Animal Science and Technology, Chung-Ang University, Anseong 456756, Korea. Tel: +82-31-670-3027, Fax: +82-31-676-5986, Email: kimgeun@cau.ac.kr sella, Streptococcus, and Bifidobacterium.

Macrophages are tissue-based phagocytes derived from monocytes that play a central role in the initiation of first line immune defense (Chon et al., 2009). Lipopolysaccharide (LPS) of gram-negative bacteria and lipoteichoic acid (LTA) of gram-positive bacteria can induce macrophage activation. Activated macrophages induce immune responses. Nitric oxide (NO) is generated via oxidation of L-arginine to L-citrulline by NO synthase (NOS) (Lee et al., 2010). During an inflammatory response, activated macrophages secrete large amounts of NO via inducible NOS (iNOS) stimulated by inflammatory signals such as pro-inflammatory cytokines and endotoxins (LPS) from several cell types (Korhonen et al., 2002). It has been reported that NO synthesized in activated macrophages can function as a mediator of numerous tumoricidal and antimicrobial effects (Lorsbach et al., 1993).

Recently, numerous studies have focused on modulating the composition of the intestinal microbiota and probiotic LAB strain-mediated inhibition of inflammatory 
responses. In vitro studies using murine RAW 264.7 macrophages and human HT-29 cells have shown that Lactobacillus plantarum KFCC11389P (Chon et al., 2009), L. rhamnosus GG (Lee et al., 2012), L. casei MCL (Choi et al., 2012), and L. casei LC-EAS (Karthikeyan et al., 2013) have immunomodulatory effects. In addition, based on the results of studies in an in vivo colitis mouse model, anti-inflammatory effects have been reported for $L$. salivarius 433118 (Feighery et al., 2008), L. plantarum HY115, L. brevis HY7401 (Lee et al., 2008), and L. plantarum Lp91 (Duary et al., 2011).

Two of the most important selection criteria for potential probiotic candidate strains are the ability to adhere to the intestinal epithelial surface and colonize the gastrointestinal (GI) tract (Byrd and Bresalier, 2004; Ouwehand and Salminen, 2003; van Tassell and Miller, 2011). Many studies have focused on alternative in vitro models to screen probiotic strains for these abilities, including using propagated human intestinal cells, such as HT-29 and Caco-2 cells, and mucin-adhesion assays (Carasi et al., 2014; Duary et al., 2011; Valeriano et al., 2014). Recently, a Caenorhabditis elegans surrogate in vivo model has been successfully used for simple, rapid, and economic high-throughput screening of potential probiotic bacteria (Park et al., 2014). The practical advantages of using this model system for screening are that the body of C. elegans is transparent, which allows clear observation of all cells in mature animals and at all developmental stages (Leung et al., 2008) and that its intestinal cells are similar in structure to those in humans (McGhee, 2007).

In this study, we investigated the ability of selected probiotic candidate strains to mediate anti-inflammatory activities in cultures of murine RAW 264.7 macrophages. Selected strains exhibiting high levels of NO production inhibition activity in LPS-stimulated RAW macrophages were identified, and their probiotic properties were further characterized using in vitro assays and a C. elegans model. Finally, we studied the milk-fermenting ability of the selected strains to assess their suitability as probiotic strains for functional fermented milk production.

\section{Materials and Methods}

\section{Selection of strains from healthy adult feces}

As an appraisal for the application of new probiotic cultures, many strains were isolated from healthy human feces using Rogosa SL agar as a selective medium (Choi et al., 2012). Typical colonies on the agar plates were selected randomly and subcultured in MRS broth, and pure cultures were obtained by restreaking on MRS agar. Basic characterization studies, such as Gram staining and catalase tests, were performed. All isolated LAB strains were maintained in MRS broth containing 25\% glycerol at -80 ${ }^{\circ} \mathrm{C}$ until use.

\section{Isolate identification}

The bacterial 16S rRNA genes were amplified by polymerase chain reaction (PCR) using universal primers 27F (5-AGAGTTTGATCMTGGCTCAG-3) and 1492R (5-G GYTACCTTGTTACGACTT-3) in a LifeTouch thermal cycler (Alpha Laboratories, UK). The cycling program was as follows: denaturation at $95^{\circ} \mathrm{C}$ for $5 \mathrm{~min}$, followed by 30 cycles of $95^{\circ} \mathrm{C}$ for $30 \mathrm{~s}, 47^{\circ} \mathrm{C}$ for $30 \mathrm{~s}$, and $72^{\circ} \mathrm{C}$ for $60 \mathrm{~s}$, with a final extension at $72^{\circ} \mathrm{C}$ for $10 \mathrm{~min}$. The PCR products were purified with a PCR purification kit (Qiagen, USA) and sequenced by Solgent (Korea).

\section{Macrophage cell culture}

RAW 264.7 cells were obtained from the Korean Cell Line Bank (Seoul, Korea). The cells were grown in Dulbecco's modified Eagle's medium (DMEM; Gibco, USA) supplemented with $10 \%$ fetal bovine serum (FBS; Gibco) and $1 \%$ streptomycin and penicillin at $37^{\circ} \mathrm{C}$ in a humidified $5 \% \mathrm{CO}_{2}$ incubator. Cell numbers and viability were measured by using Trypan blue (Amresco, USA) and a hemocytometer. Confluent cells were subcultured every 2 $\mathrm{d}$ as described previously (Choi et al., 2012).

\section{Nitric oxide assay}

RAW 264.7 cells were placed into 24 -well plates at a density of $1.0 \times 10^{5}$ cells/well. LPS $(100 \mathrm{ng} / \mathrm{mL})$ and LAB fractions (at $2.5 \%$ final concentration, 10 times concentrated) were added. After $24 \mathrm{~h}$, the concentration of NO was determined by measuring the amount of nitrite in the cell culture supernatant using Griess reagent (Sigma, USA) as previously described (Green et al., 1982). An aliquot of the culture supernatant $(50 \mu \mathrm{L})$ was mixed with an equal volume of Griess reagent and incubated for $15 \mathrm{~min}$ at room temperature. The absorbance at $540 \mathrm{~nm}$ was measured using a microplate reader. The nitrite concentration was determined based on a standard curve prepared using sodium nitrite (Kim et al., 2007).

\section{Acid tolerance}

As described previously (Hedin et al., 2000), bacterial cultures (initial density, $10^{7} \mathrm{CFU} / \mathrm{mL}$ ) were incubated in MRS broth overnight at $37^{\circ} \mathrm{C}$. The overnight cultures were divided into two equal aliquots. To measure acid tol- 
erance, bacterial cells harvested from one aliquot were suspended in MRS broth containing $0.05 \% \mathrm{~L}$-cystein $\cdot \mathrm{HCl}$ adjusted to $\mathrm{pH} 3.0$ (by adding $4 \mathrm{M} \mathrm{HCl}$ ) and incubated for $3 \mathrm{~h}$ at $37^{\circ} \mathrm{C}$. Cells harvested from the second culture aliquot were used as a control. After incubation, the acidic culture was neutralized by adding $0.1 \mathrm{M}$ PBS ( $\mathrm{pH}$ 6.2), 10-fold serial dilutions were made. Live cells were counted on MRS agar plates after incubation for $48 \mathrm{~h}$ at $37^{\circ} \mathrm{C}$. Acid tolerance was expressed as a percentage of the number of viable cells after incubation in MRS broth ( $\mathrm{pH}$ 3.0) relative to that of the control and was calculated using the following equation:

Acid tolerance $(\%)=(\log \mathrm{CFU} / \mathrm{mL}$ after $3 \mathrm{~h}$ of exposure) / (initial Log CFU/mL) $\times 100$

\section{Bile tolerance}

Bile tolerance was assessed according to the method of Gilliland et al. (1990). Briefly, MRS broth and MRS broth supplemented with $0.3 \%$ oxgall were inoculated with an overnight culture $(0.5 \%$ initial inoculum). After $12 \mathrm{~h}$ of culture at $37^{\circ} \mathrm{C}$, the optical density at $620 \mathrm{~nm}\left(\mathrm{OD}_{620}\right)$ was measured. Bile resistance was expressed as a percentage of the number of viable cells after incubation in MRS broth with oxgall compared to that of the control (without oxgall) and was calculated using the following equation:

Bile resistance $(\%)=\left(\mathrm{OD}_{620}\right.$ in MRS broth with oxgall $)$ $/\left(\mathrm{OD}_{620}\right.$ in MRS broth without oxgall $) \times 100$

\section{Hydrophobicity}

Cell surface hydrophobicity was determined using the method of Valeriano et al. (2014). Briefly, an overnight culture was harvested (13,000 rpm, $15 \mathrm{~min})$, washed twice, and resuspended in PBS to a final $\mathrm{OD}_{600}$ of $\sim 0.5$, which was designated as $\mathrm{A}_{0}$. An aliquot $(3 \mathrm{~mL})$ of the cell suspension was mixed with $1 \mathrm{~mL}$ of toluene. The mixture was mixed vigorously for $90 \mathrm{~s}$ and then left to stand at $37^{\circ} \mathrm{C}$ for $1 \mathrm{~h}$ to allow phase separation. After the toluene phase was removed, the $\mathrm{OD}_{600}$ of the aqueous phase was measured and designated as $\mathrm{A}_{1}$. Each experiment was performed in triplicate from independent cultures. Cell surface hydrophobicity was determined as the percentage decrease in the absorbance of the aqueous phase after exposure to toluene, which was calculated using the following equation (Valeriano et al., 2014):

Hydrophobicity $(\%)=\left[1-\left(\mathrm{A}_{1} / \mathrm{A}_{0}\right)\right] \times 100$

where,

$\mathrm{A}_{0}: \mathrm{OD}_{600}$ value of the original suspension
$\mathrm{A}_{1}: \mathrm{OD}_{600}$ value of the aqueous phase after extracting with toluene

\section{C. elegans intestine adhesion assay}

The $C$. elegans strain used in this study was CF512 fer15(b26)II;fem-1(hc17)IV (Kim and Mylonakis, 2012). This $C$. elegans strain was routinely maintained according to the method described by Brenner (1974) using nematode growth medium (NGM) plates seeded with Escherichia coli OP50. The isolated LAB strains were screened for colonization of the $C$. elegans intestinal tract according to a previously described method (Park et al., 2014). Briefly, after exposing C. elegans to individual LAB strains on NGM plates containing nystatin for $5 \mathrm{~d}, 10$ worms were randomly selected, washed twice with M9 buffer, and place on brain-heart infusion (BHI) plates containing kanamycin and streptomycin. These plates then were exposed to gentamycin $(5 \mu \mathrm{L}$ of a $25 \mu \mathrm{g} / \mathrm{mL}$ solution) for $5 \mathrm{~min}$. After the worms were washed three times with M9 buffer, they were pulverized using a pestle (Kontes Glass Inc., USA) in a 1.5-mL Eppendorf tube containing M9 buffer supplemented with 1\% Triton X-100. The worm lysate was serially diluted (10-fold) in M9 buffer and plated on MRS agar ( $\mathrm{pH}$ 5.0). After incubation for 48 $\mathrm{h}$ at $37^{\circ} \mathrm{C}$, live bacterial cells in the lysates were counted.

\section{Culture characteristics in skim milk}

The cell viability, $\mathrm{pH}$, and titratable acidity (TA) of the LAB selected strains and L. casei YIT 9029 (obtained from Korea Yakult Co.) were assessed during growth in skim milk media (Choi et al., 2012). Overnight cultures were containing $10 \%$ skim milk and $2 \%$ glucose were incubated at $37^{\circ} \mathrm{C}$ in a water bath. Viable cells were counted at $4 \mathrm{~h}$ intervals for $36 \mathrm{~h}$. Cultured cells were serially diluted in PBS and then plated on MRS agar. Plates were incubated at $37^{\circ} \mathrm{C}$ for $48 \mathrm{~h}$. The $\mathrm{pH}$ was measured using a $\mathrm{pH}$ meter $\left(\right.$ Seven easy ${ }^{\mathrm{TM}}$ S20; Mettler Toledo, USA). To measure the TA, the cultured samples were titrated with $0.1 \mathrm{~N} \mathrm{NaOH}$. TA was expressed as the percentage of lactic acid contents in the sample.

\section{Results and Discussion}

\section{Screening and identification of isolates}

Molecular characterization of 24 fecal isolates using $16 \mathrm{~S}$ rDNA sequencing analysis (Table 1 ) showed that $L$. plantarum (6 strains) was the most common species, followed by L. sakei subsp. sakei (4 strains), Pediococcus pentosaceus (3 strains), L. curvatus (2 strains), L. salivar- 
Table 1. 16S r DNA sequence homology with the type strains obtained from Ez-taxon search

\begin{tabular}{|c|c|c|c|}
\hline Strain ID & Identified as & Type strain ${ }^{\mathrm{a}}$ & "Homology (\%) \\
\hline 1 & Lactobacillus plantarum CAU1045 & ATCC $14917^{T}$ & 99.27 \\
\hline 2 & Lactobacillus paracasei subsp. tolerans CAU1048 & JCM $1171^{\mathrm{T}}$ & 99.38 \\
\hline 3 & Lactobacillus plantarum CAU1054 & ATCC $14917^{\mathrm{T}}$ & 99.36 \\
\hline 4 & Lactobacillus plantarum CAU1055 & ATCC $14917^{\mathrm{T}}$ & 99.81 \\
\hline 5 & Lactobacillus plantarum CAU1064 & ATCC $14917^{\mathrm{T}}$ & 99.14 \\
\hline 6 & Streptococcus infantarius subsp. coli CAU1085 & NCDO $964^{\mathrm{T}}$ & 99.93 \\
\hline 7 & Lactobacillus plantarum CAU1106 & ATCC $14917^{\mathrm{T}}$ & 99.68 \\
\hline 8 & Pediococcus pentosaceus CAU1212 & DSM $20336^{\mathrm{T}}$ & 99.06 \\
\hline 9 & Lactobacillus acidophilus CAU1214 & ATCC $4356^{\mathrm{T}}$ & 99.72 \\
\hline 10 & Pediococcus pentosaceus CAU1216 & DSM $20336^{\mathrm{T}}$ & 99.31 \\
\hline 11 & Weissella cibaria CAU1221 & KACC $11862^{\mathrm{T}}$ & 99.44 \\
\hline 12 & Weissella viridescens CAU1222 & NRIC $1536^{\mathrm{T}}$ & 99.93 \\
\hline 13 & Pediococcus pentosaceus CAU1223 & DSM $20336^{\mathrm{T}}$ & 99.32 \\
\hline 14 & Weissella viridescens CAU1224 & NRIC $1536^{\mathrm{T}}$ & 98.39 \\
\hline 15 & Weissella cibaria CAU1225 & KACC $11862^{\mathrm{T}}$ & 99.52 \\
\hline 16 & Lactobacillus sakei subsp. sakei CAU1241 & DSM $20017^{\mathrm{T}}$ & 99.86 \\
\hline 17 & Lactobacillus sakei subsp. sakei CAU1242 & DSM $20017^{\mathrm{T}}$ & 99.78 \\
\hline 18 & Lactobacillus curvatus CAU1243 & LMG $9198^{\mathrm{T}}$ & 99.64 \\
\hline 19 & Lactobacillus curvatus CAU1244 & LMG $9198^{\mathrm{T}}$ & 99.64 \\
\hline 20 & Lactobacillus sakei subsp. sakei CAU1245 & DSM $20017^{\mathrm{T}}$ & 99.86 \\
\hline 21 & Lactobacillus sakei subsp. sakei CAU1273 & DSM $20017^{\mathrm{T}}$ & 99.90 \\
\hline 22 & Lactobacillus salivarius CAU1301 & ATCC $11741^{\mathrm{T}}$ & 99.86 \\
\hline 23 & Lactobacillus salivarius CAU1302 & ATCC $11741^{\mathrm{T}}$ & 99.86 \\
\hline 24 & Lactobacillus plantarum CAU1364 & ATCC $14917^{\mathrm{T}}$ & 99.81 \\
\hline
\end{tabular}

${ }^{a}$ ATCC, American Type Culture Collection; DSM, Deutsche Sammlung von Mikroorganismen; JCM, Japan Collection of Microorganisms; KACC, Korean Agricultural Culture Collection; LMG, LMG Bacteria Collection Universiteit Gent; NCDO, National Collection of Dairy Organism; NRIC, NODAI Research Institute Culture Collection

ius (2 strains), Weissella cibaria (2 strains), W. viridescens (2 strains), L. paracasei subsp. tolerans (1 strain), L. acidophilus (1 strain), and Streptococcus infantarius subsp. coli (1 strain).

\section{Nitric oxide assay}

Initially, the anti-inflammatory activity of the LAB isolates was investigated in LPS-stimulated RAW 264.7 cells. Heat-killed whole bacterial cells of the isolated LAB strains were added to the RAW cell cultures, and inhibition of NO production was evaluated. NO is a suspected mediator in the development of diseases associated with chronic inflammation, including cancer and inflammatory bowel disease. NO also has various biological functions in many types of immune cells, including induction of the bactericidal effect in macrophages and signal transduction during inflammation (Jeong et al., 2010). The ability of the LAB strains to inhibit NO production was expressed as a relative NO rate (\%) compared with the NO concentration in the controls (Fig. 1). The positive RAW 264.7 cell control culture was treated with LPS only, and the negative control was treated with PBS. As shown in Fig.
2 , potential anti-inflammatory effects were observed in cultures with 8 isolated LAB strains (CAU1054, CAU 1055, CAU1064, CAU1106, CAU1245, CAU1273, CAU 1301, and CAU1302) belonging to the genus Lactobacillus. Four L. plantarum strains reduced NO production; however, the other 2 L. plantarum strains increased NO production in the RAW cell cultures, suggesting that the anti-inflammatory effects are strain specific, even within the same species. In addition, some strains of L. acidophilus, L. sakei subsp. sakei, and L. salivarius also inhibited NO production. However, other LAB strains, including $L$. paracasei, S. infantarius subsp. coli, P. pentosaceus, W. cibaria, W. viridescens, and L. curvatus increased NO production. According to Chon et al. (2009), intracellular metabolites or molecules from L. plantarum KFCC11389P have inhibitory effects on the production of the proinflammatory cytokines IL-6 and TNF- $\alpha$ in LPS-stimulated RAW 264.7 macrophages. However, these antiinflammatory effects were not detected when viable or heat-killed cells were used, suggesting that the L. plantarum KFCC11389P cells did not affect the anti-inflammatory cytokines, and thus could not exert the immune- 


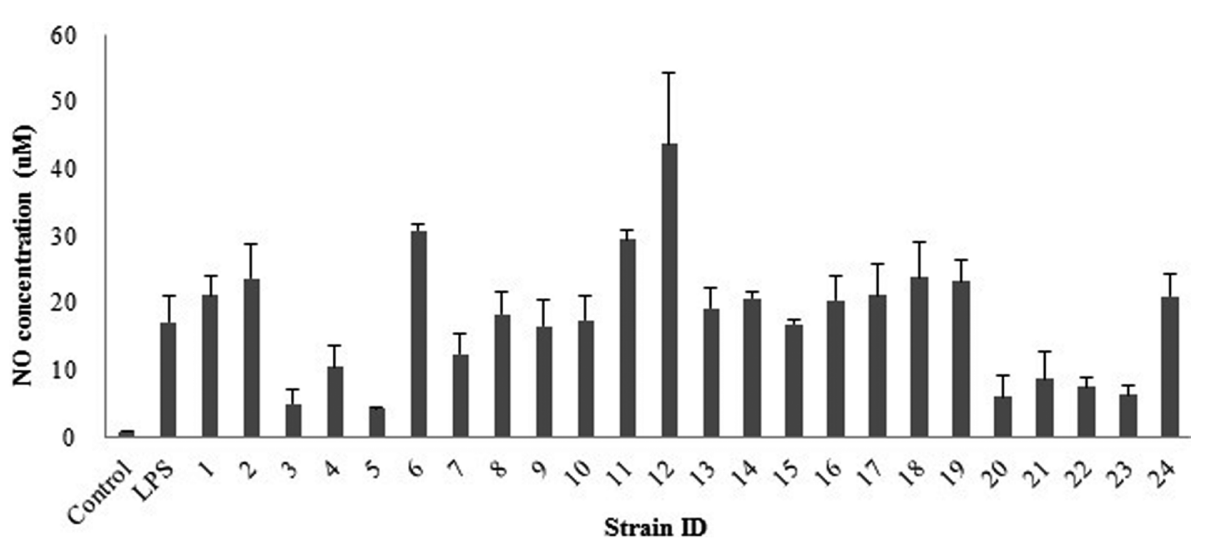

Fig. 1. Nitric oxide concentration $(\mu \mathrm{M})$ in the RAW 264.7 cells treated with the heat killed whole cells of 24 LAB isolates (Strain ID: See the Table 1 for the identification of the isolates).

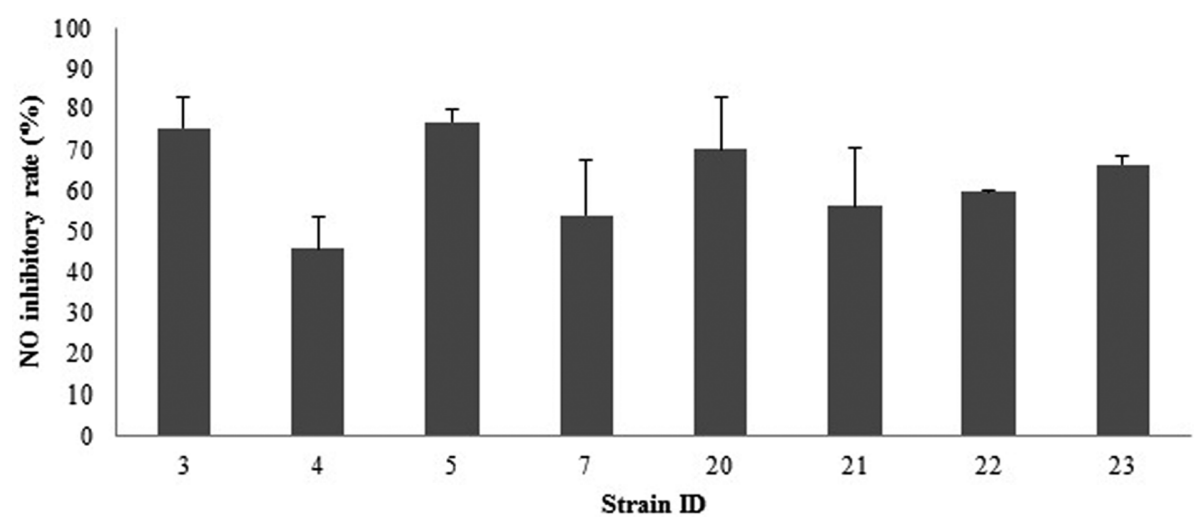

Fig. 2. Comparison of inhibitory rate (\%) in NO production in the RAW 264.7 cells treated with the heat killed whole cells of 8 selected LAB strains (Strain ID: 3. L. plantarum CAU1054; 4. L. plantarum CAU1055; 5. L. plantarum CAU1064; 7. L. plantarum CAU1106; 20. L. sakei subsp. sakei CAU1245; 21. L. sakei subsp. sakei CAU1273; 22. L. salivarius CAU1301; 23. L. salivarius CAU1302).

modulating activities. Karthikeyan et al. (2013) reported that a solvent fraction extracted from a strain of $L$. casei (LC-EAS) can inhibit NO production by suppressing iNOS mRNA expression.

To obtain more insight into the anti-inflammatory effects exerted by selected L. plantarum strains, future studies are be needed to identify the active components in the bacterial cell structure and to verify the anti-inflammatory activity in animal models of disease and clinical experiments in humans.

\section{Acid tolerance}

Survival of probiotics during gastric transit is important for colonization of the GI tract. Therefore, resistance to low $\mathrm{pH}$ was examined as a first step in determining the probiotic potential of the LAB strains (McDonald et al., 1990). The survival rate of the LAB strains was investi- gated after $3 \mathrm{~h}$ of incubation in MRS broth at $\mathrm{pH}$ 3.0. As shown in Fig. 3, 17 strains showed survival rates above $90 \%$ after $3 \mathrm{~h}$ of incubation at low $\mathrm{pH}$. Among these acid tolerant strains, 3 strains of L. plantarum (CAU1054, CAU1055, and CAU1064) and L. salivarius CAU1301 showed higher survival than the other strains (Fig. 3). In contrast, the viable counts of the $S$. infantarius subsp. coli, $W$. cibaria, L. curvatus, and L. sakei subsp. sakei strains were less than $1 \log \mathrm{CFU} / \mathrm{mL}$ after $3 \mathrm{~h}$ of exposure to $\mathrm{pH}$ 3.0.

Several in vitro assays have been developed for the selection of acid-resistant LAB strains, including incubation in gastric contents, exposure to $\mathrm{pH}$-adjusted PBS, and the use of a dynamic model of the stomach (Alander et al., 1999). The acid tolerance test (conducted at $\mathrm{pH}$ 3.0) used in this study has been shown to be sufficient for screening acid-tolerant strains from many LAB isolates. Further- 


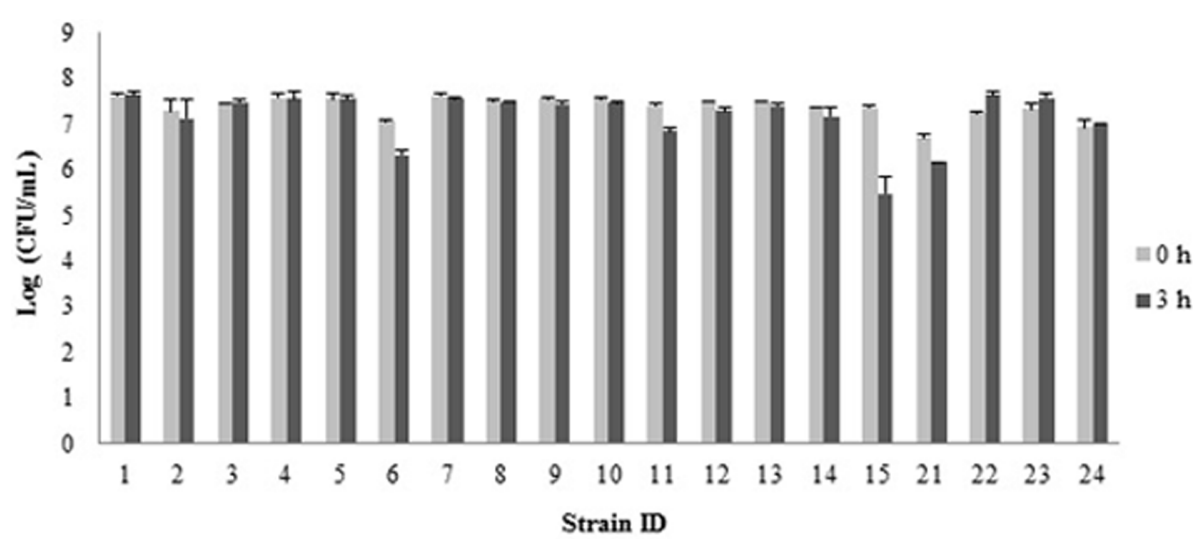

Fig. 3. Survival rates of lactic acid bacteria after $3 \mathrm{~h}$ exposure to $\mathrm{pH} 3.0$ in MRS broth (Strain ID: See the Table 1 for the identification of the isolates). Data are an average of the data obtained by three independent experiments.

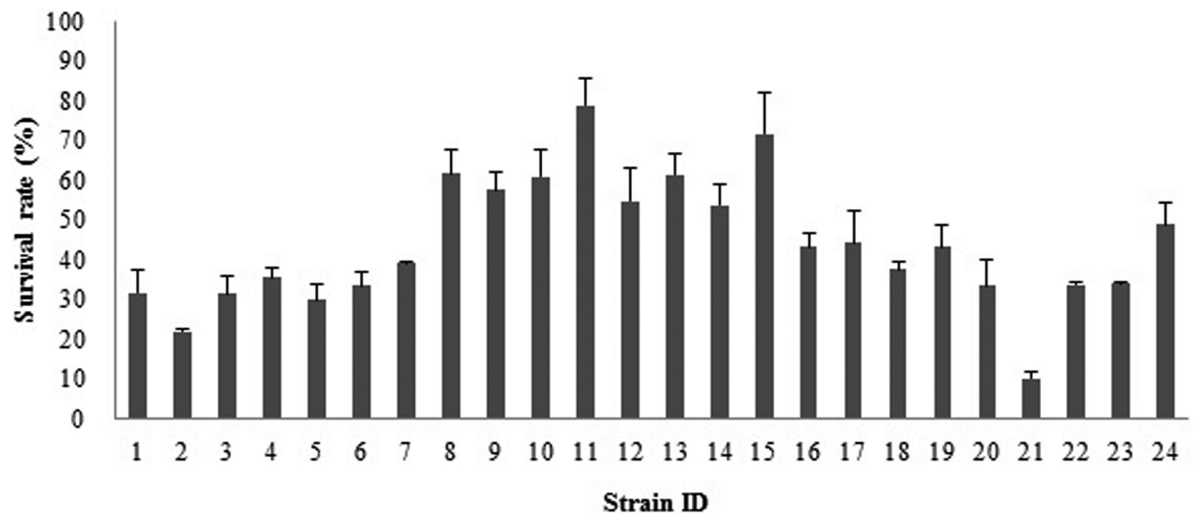

Fig. 4. Survival rates of the lactic acid bacteria after $12 \mathrm{~h}$ in MRS broth with $0.3 \%$ oxgall (Strain ID: See the Table 1 for the identification of the isolates).

more, Argyri et al. (2013) postulated that a $\mathrm{pH}$ value of 2.5 for the selection of potential probiotic strains is very selective because it is not the most common $\mathrm{pH}$ in the human stomach due to the buffering capacity of food or other carrier matrix molecules following consumption.

\section{Bile tolerance}

Bile salt tolerance is considered one of the properties required for the survival of probiotic strains in the small intestine. Bile secreted into the small intestine plays an important role in lipid digestion. However, due its amphiphilic nature, it is somewhat toxic to the GI microbiota because it can damage the lipid bilayer of bacterial cell membranes. The concentration of bile used here, $0.3 \%$, is considered to be the critical concentration for screening resistant strains (Hyronimus et al., 2000). As shown in Fig. 4, P. pentosaceus, L. acidophilus, W. cibaria, $W$. viridescens strains showed bile tolerance, whereas 1 strain of $L$. sakei subsp. sakei was the most susceptible to $0.3 \%$ oxgall. The bile tolerance of the selected LAB strains appears to be a strain-specific characteristic, as was reported previously (Mishra and Prasad, 2005).

\section{Hydrophobicity}

Bacterial cell surface hydrophobicity is important for the interactions between the bacterium and host intestinal epithelial cells that initiate bacterial adhesion in the GI tract. Previously, Perez et al. (1998) reported a high correlation between the hydrophobicity of the bacterial cell surface and adherence to intestinal epithelial cells. In the in vitro hydrophobicity test (Fig. 5), 7 strains showed greater than $50 \%$ hydrophobicity; 6 of them were members of the genus Lactobacillus and the remaining strain, 224, was identified as $W$. viridescens. These results are in a good agreement with those of Valeriano et al. (2010), who reported that all tested Lactobacillus strains showed 


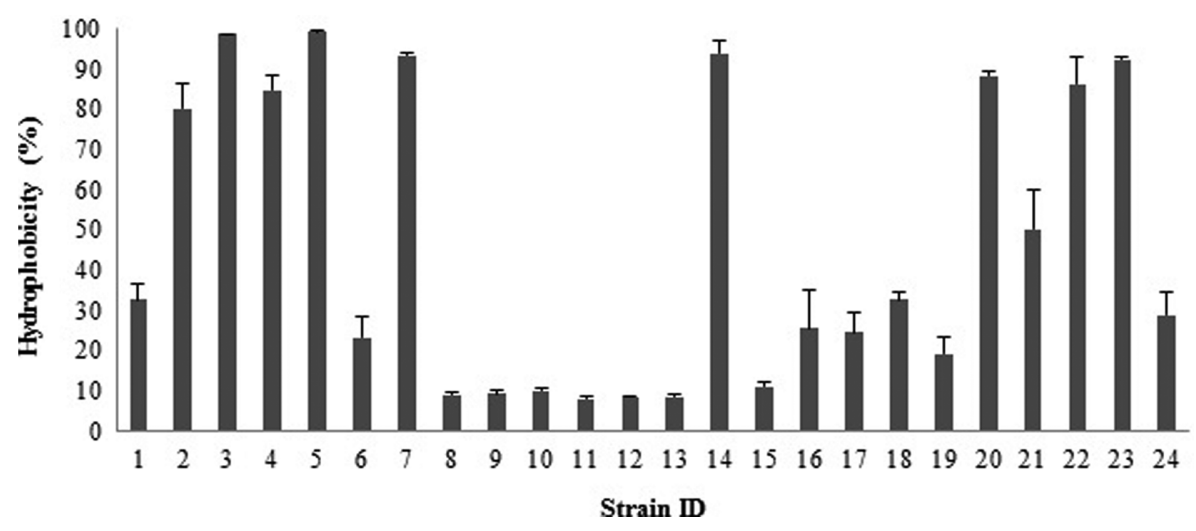

Fig. 5. Comparison of in vitro hydrophobicity of LAB isolates (Strain ID: See the Table 1 for the identification of the isolates).

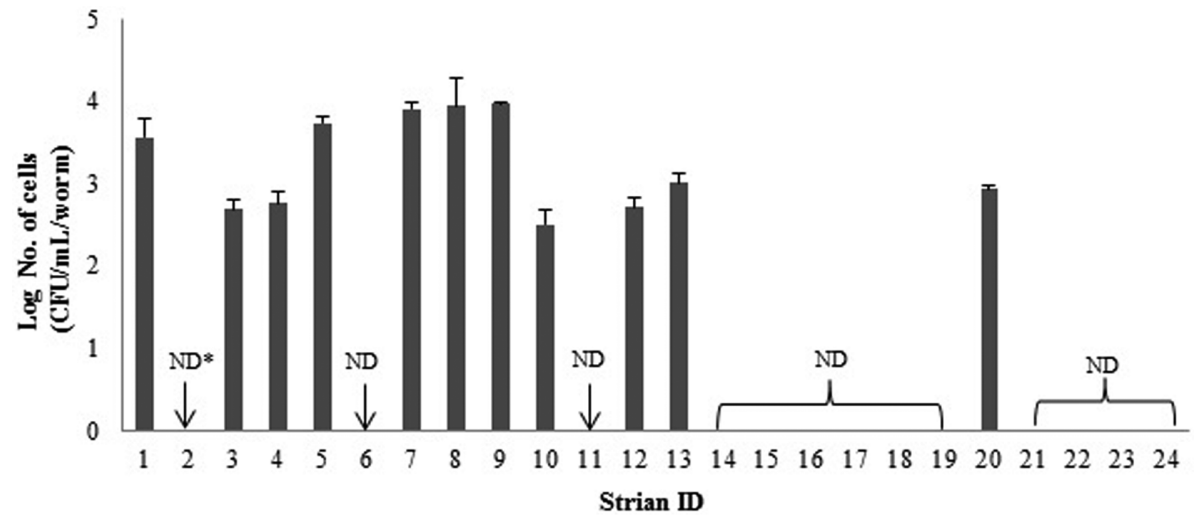

Fig. 6. Colonization of lactic acid bacteria on the $C$. elegans intestine (*Not detected) (Strain ID: See the Table 1 for the identification of the isolates).

hydrophobic surface characteristics ( $>50 \%$ ), whereas some gram-negative bacteria, such as E. coli and Salmonella Typhimurium, exhibited relatively hydrophilic surfaces. Valeriano et al. (2014) also reported a significant correlation between in vitro adhesion on mucin, bacterial cell surface hydrophobicity, and auto-aggregation for the $L$. mucosae LM1 strain. However, these in vitro data may not correlate with the in vivo conditions due to various strain-specific mechanisms. Although some alternative in vitro adhesion assay models of the epithelial surface have been described, they have a critical limitation with regard to reflecting the actual human intestine.

\section{C. elegans intestine adhesion}

C. elegans is an accepted in vivo model to study bacteria-host interactions in the gut because the intestinal cells of this nematode worm are similar to those of humans (Park et al., 2014). Therefore, we used C. elegans as a surrogate in vivo screening system for potential probiotic
LAB with the ability to adhere to the intestinal tract. As shown in Fig. 6, 5 strains (CAU1045, CAU1064, CAU 1106, CAU1212, and CAU1214) showed relatively high GI tract colonization. These strains exhibited outstanding persistence in the C. elegans intestine ( $>3.5 \mathrm{Log} \mathrm{CFU} / \mathrm{mL}$ per worm). Another 6 strains (CAU1054, CAU1055, CAU1216, CAU1222, CAU1223, and CAU1245) also showed good colonization ability (2.5-3.0 Log CFU/mL per worm). However, the remaining strains were not able to colonize the intestinal tract of $C$. elegans. The results of the present study showed that in vivo colonization ability was a strain-specific characteristic. For example, $5 \mathrm{~L}$. plantarum strains exhibited high colonization, whereas 1 L. plantarum strain (CAU1364) did not shown high colonization. Park et al. (2014) also reported that 4 strains of L. plantarum exhibited good colonizing ability, and these strains significantly extended the life span of C. elegans and enhanced their resistance to pathogenic bacteria such as $S$. aureus. It would be interesting to investigate the cor- 


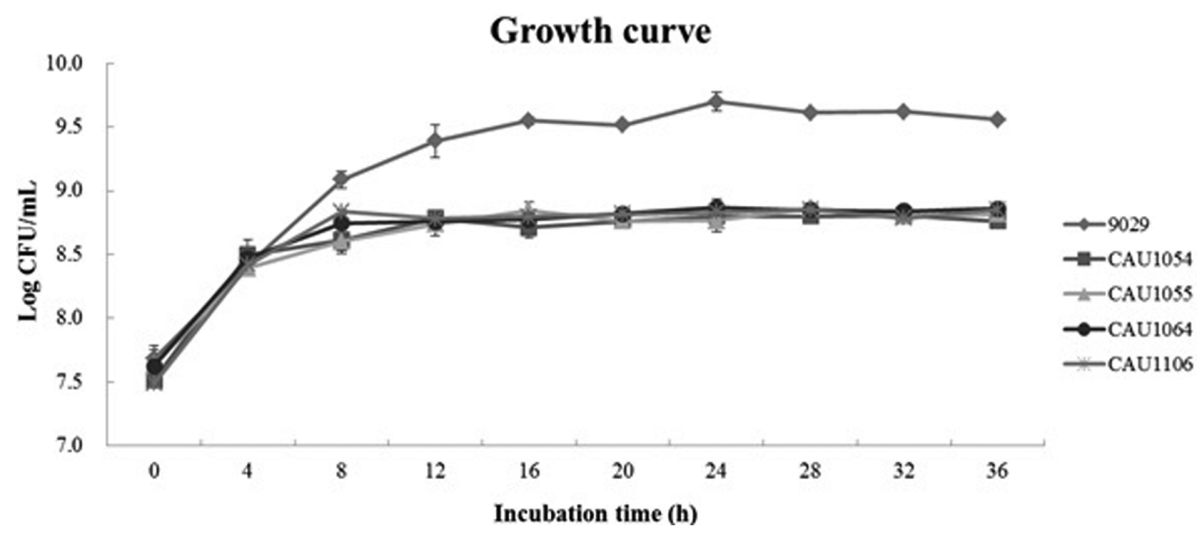

Fig. 7. Viable cell count of the Lactobacillus plantarum strains during the growth in $10 \%$ skim milk supplemented $2 \%$ glucose.
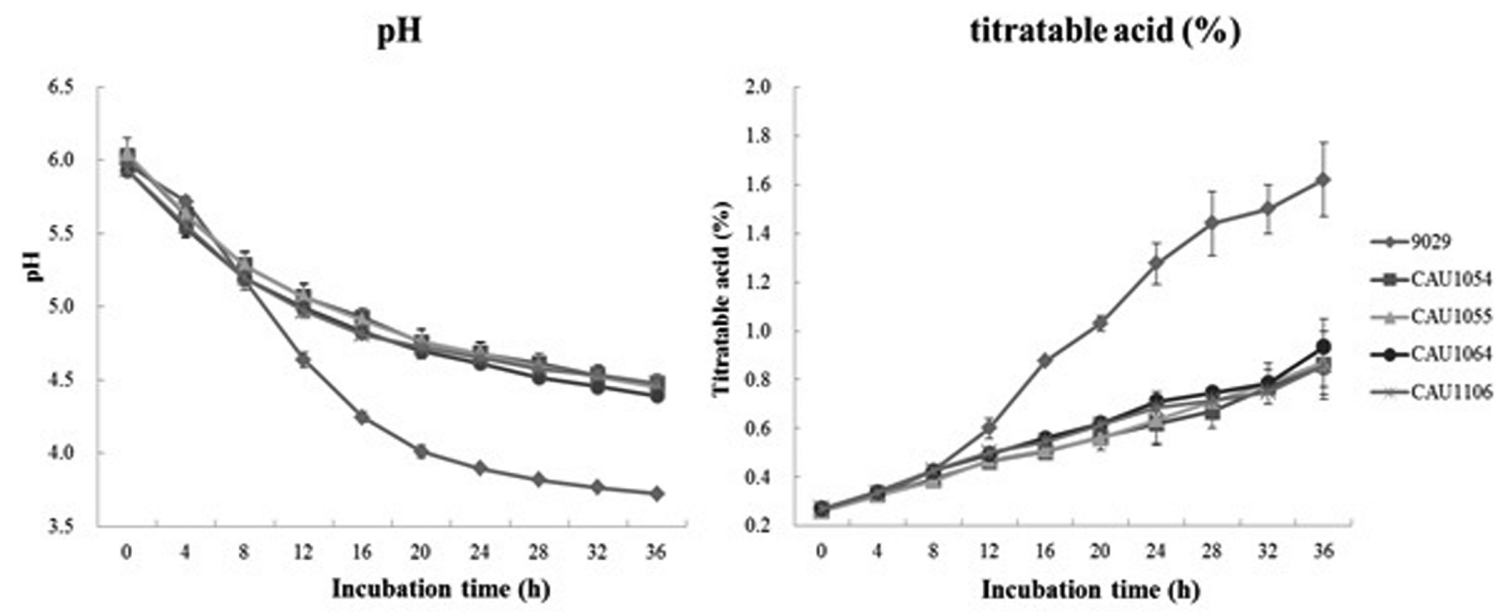

Fig. 8. The change of $\mathrm{pH}$ and titratable acidity of $10 \%$ skim milk supplemented with $2 \%$ glucose.

relation between colonizing ability and anti-inflammatory activity using good-colonizing and poor-colonizing $L$. plantarum strains in a future study.

\section{Skim milk culture characteristics}

Yogurt or fermented milk is one of the most efficient probiotic carrier foods. The milk fermenting ability of LAB strains is very important for use as a starter culture in dairy production. We investigated the growth characteristics of the $L$. plantarum strains that showed excellent anti-inflammatory activity (CAU1054, CAU1055, CAU1064, and CAU1106) in skim milk media. The selected strains were able to grow in skim milk media, reaching viable cell counts of $8.5 \mathrm{Log} \mathrm{CFU} / \mathrm{mL}$ (Fig. 7), which was lower than that of L. casei YIT 9029 (also known as strain Shirota). Accordingly, the $\mathrm{pH}$ and TA values of the selected skim milk cultures were lower than those of $L$. casei YIT 9029 (Fig. 8). In order to use these probiotic candidates for yogurt production, it would be necessary to test for potential growth promoting conditions in milk and/or to co-culture them with other starter bacteria. Alternatively, these strains could be used in other probiotic applications with efficient delivery systems, including as lyophilized powders, capsules, and tablets.

\section{Conclusions}

We investigated the ability of selected probiotic candidate strains reduce NO production levels in cultures of LPS-stimulated RAW 264.7 macrophages. Based on morphological, biochemical, and molecular biological characterizations, the selected strains were identified as $L$. plantarum. The $4 \mathrm{~L}$. plantarum strains isolated and characterized in this study meet the probiotic selection criteria for in vitro probiotic property assessment. Furthermore, they showed good colonization in a $C$. elegans intestine 
in vivo model. Taken together, the selected L. plantarum strains show promise for use as probiotic agents and in fermented dairy food applications. Further studies are needed to obtain more insight into the anti-inflammatory effects of these strains in animal models and human clinical experiments.

\section{Acknowledgements}

This research was supported by the Chung-Ang University Excellent Student Scholarship in 2014 and funded by a grant from the Korea Food Research Institute (project no. E0131901).

\section{References}

1. Alander, M., De Smet I., Nollet, L., Verstraete, W., von Wright, A., and Mattila-Sandholm, T. (1999) The effect of probiotic strains on the microbiota of the simulator of the human intestinal microbial ecosystem (SHIME). Int. J. Food. Microbiol. 46, 71-79.

2. Argyri, A. A., Zoumpopoulou, G., Karatzas, K. A. G., Tsakalidou, E., Nychas, G. J. E., Panagou, E. Z., and Tassou, C. C. (2013) Selection of potential probiotic lactic acid bacteria from fermented olives by in vitro tests. Food Microbiol. 33, 282-291.

3. Brenner, S. (1974) The genetics of Caenorhabditis elegans. Genetics 77, 71-94.

4. Byrd, J. C. and Bresalier, R. S. (2004) Mucins and mucin binding proteins in colorectal cancer. Cancer Metastasis Rev. 23, 77-99.

5. Carasi, P., Ambrosis, N. M., De Antoni, G. L., Bressollier, P., Urdaci, M. C., and Serradell Mde, L. (2014) Adhesion properties of potentially probiotic Lactobacillus kefiri to gastrointestinal mucus. J. Dairy Res. 81, 16-23.

6. Choi, J. K., Lim, Y. S., Kim, H. J., Hong, Y. H., Ryu, B. Y., and Kim, G. B. (2012) Screening and characterization of Lactobacillus casei MCL strain exhibiting immunomodulation activity. Korean J. Food Sci. An. 32, 635-643.

7. Chon, H., Choi, B., Lee, E., and Jeong, G. (2009) Immunomodulatory effects of specific bacterial component of Lactobacillus plantarum KFCC11389P on the murine macrophage cell line RAW 264.7. J. Appl. Microbiol. 107, 1588-1597.

8. Duary, R. K., Rajput, Y. S., Batish, V. K., and Grover, S. (2011) Assessing the adhesion of putative indigenous probiotic lactobacilli to human colonic epithelial cells. Indian $J$. Med. Res. 134, 664-671.

9. Feighery, L. M., Smith, P., O’Mahony, L., Fallon, P. G., and Brayden, D. J. (2008) Effects of Lactobacillus salivarius 433 118 on intestinal inflammation, immunity status and in vitro colon function in two mouse models of inflammatory bowel disease. Dig. Dis. Sci. 53, 2495-1506.

10. Food and Agriculture Organization of the United Nations. (2001) Health and nutritional properties of probiotics in food including powder milk with live lactic acid bacteria. (http:// www.who.int/foodsafety/publications/fs_management/en/pr obiotics/pdf)

11. Gilliland, S. E. and Walker, D. K. (1990) Factors to consider when selecting a culture of Lactobacillus acidophilus as a dietary adjunct to produce a hypocholesterolemic effect in human. J. Dairy Sci.73, 905-911.

12. Green, L. C., Wagner, D. A., Glogowski, J., Skipper, J., Wishnok, J. S., and Tannenbaum, S. R. (1982) Analysis of nitrate, nitrite, and $[15 \mathrm{~N}]$ nitrate in biological fluids. Anal. Biochem. 126, 131-138.

13. Hedin, C., Whelani, K., and Lindsay, J. O. (2000) Evidence for the use of probiotics and probiotics in inflammatory bowel diseases: a review of clinical trials. Proc. Nutr. Sor. 66, 307-315.

14. Hyronimus, B., Le Marrec, C., HadjSassi, A., and Deschamps, A. (2000) Acid and bile tolerance of spore-forming lactic acid bacteria. Int. J. Food Microbiol. 61, 193-197.

15. Jeong, I. Y., Lee, H. J., Jin, C. H., Park, Y. D., Choi, D. S., and Kang, M. A. (2010) Anti-inflammatory activity of Stevia rebaudiana in LPS-induced RAW 264.7 cells. J. Food Sci. Nutr. 15, 14-18.

16. Karthikeyan, T., Pravin, M., Muthusamy, V. S., Raja, R. B., and Lakshmi, B. S. (2013) In vitro investigation of the immunomodulatory potential of probiotic Lactobacillus casei. Probiotics Antimicro. Prot. 5, 51-58.

17. Kim, D. W., Cho, S. B., Yun, C. H., Jeong, H. Y., Chung, W. T., Choi, C. W., Lee, H. J., Nam I. S., Suh, H. H., Lee, S. S., and Lee, B. S. (2007) Induction of cytokines and nitric oxide in murine macrophages stimulated with enzymatically digested Lactobacillus strains. J. Microbiol. 45, 373-378.

18. Kim, Y. and Mylonakis, E. (2012) Caenorhabditis elegans immune conditioning with the probiotic bacterium Lactobacillus acidophilus strain NCFM enhances gram-positive immune responses. Infect. Immun. 80, 2500-2508.

19. Korhonen, R., Korpela, R., and Moilanen, E. (2002) Signalling mechanisms involved in the induction of inducible nitric oxide synthase by Lactobacillus rhamnosus GG, endotoxin, and lipoteichoic acid. Inflammation 26, 207-214.

20. Lee, E. K., Lee, N. K., Lee, S. K., Chang, H. I., and Paik, H. D. (2010) Screening of immunostimulatory probiotic lactic acid bacteria from chicken feces as animal probiotics. Korean J. Food Sci. An. 30, 634-640.

21. Lee, H. S., Han, S. Y., Bae, E. A., Huh, C. S., Ahn, Y. T., Lee, J. H., and Kim, D. H. (2008) Lactic acid bacteria inhibit proinflammatory cytokine expression and bacterial glycosaminoglycan degradation activity in dextran sulfate sodium-induced colitic mice. Int. Immunopharmacol. 8, 574-580.

22. Lee, S. K., Yang, K. M., Cheon, J. H., Kim, T. I., and Kim, W. H. (2012) Anti-inflammatory mechanism of Lactobacillus rhamnosus GG in lipopolysaccharide-stimulated HT-29 cell. Korean J. Gastroenterol. 60, 86-93.

23. Leung, M. C. K., Williams, P. L., Benedetto, A., Au, C., Helmcke, K. J., Aschner, M., and Meyer, J. N. (2008) Caenorhabditis elegans: An emerging model in biomedical and environmental toxicology. Toxicol. Sci. 106, 5-28. 
24. Lorsbach, R. B., Murphy, W. J., Lowenstein, C. J., Snyder, S. H., and Russell, S. W. (1993) Expression of the nitric oxide synthase gene in mouse macrophages activated for tumor cell killing: molecular basis for the synergy between interferongamma and lipopolysaccharide. J. Biol. Chem. 268, 19081913.

25. Maldonado, G. C., Doreno, D., de LeBlanc, A., Vinderola, G., Bibas, B., Perdigo'nG, M. E. (2007) Proposed model: Mechanisms of immunomodulation induced by probiotic bacteria. Clin. Vaccine Immunol. 14, 485-492.

26. Mcdonald, L. C., Fleming, H. P., and Hassan, H. M. (1990) Acid tolerance of Leuconostoc mesenteroides and Lactobacillus plantarum. Appl. Environ. Microbiol. 56, 2120-2124.

27. McGhee, J. D. (2007) The C. elegans intestine. WormBook 27, 1-36.

28. Mishra, V. and Prasad, D. N. (2005) Application of in vitro methods for selection of Lactobacillus casei strains as potential probiotics. Int. J. Food Microbiol. 103, 109-115.
29. Ouwehand, A. C. and Salminen, S. (2003) In vitro adhesion assays for probiotics and their in vivo relevance: a review. Microb. Ecol. Health Dis. 15, 175-184.

30. Park, M. R., Yun, H. S., Son, S. J., Oh, S., and Kim, Y. (2014) Development of a direct in vivo screening model to identify potential probiotic bacteria using Caenorhabditis elegans. $J$. Dairy Sci. 97, 6828-6834.

31. Perez, P. F., Minnaard, Y., Disalvo, E. A., and Antoni, G. L. (1998) Surface properties of bifidobacterial strains of human origin. Appl. Environ. Microb. 64, 21-26.

32. Valeriano, V. D., Parungao-Balolong, M. M., and Kang, D. K. (2014) In vitro evaluation of the mucin-adhesion ability and probiotic potential of Lactobacillus mucosae LM1. J. Appl. Microbiol. 117, 485-497.

33. Van Tassell, M. L. and Miller, M. J. (2011) Lactobacillus adhesion to mucus. Nutrients 3, 613-636.

(Received 2014.11.30/Revised 2014.12.15/Accepted 2014.12.17) 